\title{
Effects of anesthetics on early postoperative cognitive outcome and intraoperative cerebral oxygen balance in patients undergoing lung surgery: a randomized clinical trial
}

\section{Effets des agents anesthésiques sur l'issue cognitive postopératoire précoce et l'équilibre peropératoire d'oxygène cérébral chez les patients subissant une chirurgie pulmonaire: une étude clinique randomisée}

\author{
Junji Egawa, MD • Satoki Inoue, MD • Tadashi Nishiwada, MD • Takashi Tojo, MD • \\ Michitaka Kimura, MD • Takeshi Kawaguchi, MD • Shigeki Taniguchi, MD • \\ Hitoshi Furuya, MD • Masahiko Kawaguchi, MD \\ Received: 18 January 2016/Revised: 31 May 2016/Accepted: 6 July 2016/Published online: 13 July 2016 \\ (C) Canadian Anesthesiologists' Society 2016
}

\begin{abstract}
Purpose One-lung ventilation (OLV) may impair cerebral oxygen balance and induce postoperative cognitive dysfunction (POCD). It is unclear whether the type of anesthetic influences the incidence of POCD in patients undergoing OLV. This prospective study compared the incidence of POCD and intraoperative cerebral oxygen desaturation in OLV patients anesthetized with propofol vs sevoflurane during lung surgery.

Methods There were 148 participants enrolled in this study and randomized equally to either the propofol or the sevoflurane group. Anesthesia was maintained with either propofol or sevoflurane combined in both groups with fentanyl and epidural anesthesia. Regional cerebral oxygen saturation $\left(\mathrm{rSO}_{2}\right)$, jugular bulb venous oxygen saturation $\left(\mathrm{SjO}_{2}\right)$, and the incidence of cerebral oxygen desaturation $\left(\mathrm{rSO}_{2}\right.$ or $\mathrm{SjO}_{2}<50 \%$ or $\mathrm{rSO}_{2}<80 \%$ of baseline) were measured during anesthesia. Cognitive
\end{abstract}

Electronic supplementary material The online version of this article (doi:10.1007/s12630-016-0700-4) contains supplementary material, which is available to authorized users.

J. Egawa, MD $(\varangle) \cdot S$. Inoue, MD · T. Nishiwada, MD .

H. Furuya, MD - M. Kawaguchi, MD

Department of Anesthesiology, Nara Medical University, 840

Shijo-cho, Kashihara, Nara 634-8522, Japan

e-mail: junji-egawa@naramed-u.ac.jp

T. Tojo, MD · M. Kimura, MD - T. Kawaguchi, MD .

S. Taniguchi, MD

Department of Thoracic and Cardiovascular Surgery, Nara

Medical University, Kashihara, Japan function was assessed using seven neurocognitive tests two days preoperatively, five days postoperatively (primary outcome), and three months postoperatively. Bivariable and multivariable regression analyses were conducted to identify factors associated with POCD.

Results Rates of POCD did not differ statistically between groups five days postoperatively (propofol, 16/72 patients; sevoflurane, 24/72 patients; RR, 0.67; 95\% CI, 0.39 to $1.15 ; P=0.14$ ) or three months postoperatively (propofol, 9/60 patients; sevoflurane, 12/58 patients; RR, 0.73; $95 \%$ CI, 0.33 to $1.59 ; P=0.42$ ). Only three subjects per group showed intraoperative cerebral oxygen desaturation. Multivariable regression analysis revealed older age as an independent predictor of POCD.

Conclusions No statistically significant difference in the incidence of POCD could be detected between the sevoflurane and propofol anesthesia groups. Postoperative cognitive dysfunction was relatively frequent following OLV in both groups. (Registration number: UMIN 000002826).

\section{Résumé}

Objectif La ventilation unipulmonaire (VUP) pourrait avoir un impact négatif sur l'équilibre d'oxygène cérébral et induire une dysfonction cognitive postopératoire (DCPO). Nous ne savons pas si le type d'agent anesthésique influence l'incidence de DCPO chez les patients recevant une VUP. Cette étude prospective a comparé l'incidence de DCPO et de désaturation peropératoire en oxygène cérébral chez les patients sous 
VUP anesthésiés avec du propofol vs du sévoflurane pendant une chirurgie pulmonaire.

Méthode Au total, 148 patients ont participé à cette étude et ont été randomisés en deux groupes égaux à recevoir $d u$ propofol ou du sévoflurane. L'anesthésie a été maintenue à l'aide de propofol ou de sévoflurane, et l'agent de choix a été combiné à du fentanyl et à une anesthésie péridurale dans les deux groupes. La saturation en oxygène cérébral régional $\left(\mathrm{rSO}_{2}\right)$, la saturation en oxygène veineux au bulbe de la veine jugulaire $\left(\mathrm{SjO}_{2}\right)$ et l'incidence de désaturation en oxygène cérébral ( $r \mathrm{SO}_{2}$ ou $\mathrm{SjO}_{2}<50 \%$ ou $\mathrm{rSO}_{2}<80 \%$ par rapport aux valeurs de base) ont été mesurées pendant l'anesthésie. La fonction cognitive a été évaluée à l'aide de sept tests neurocognitifs deux jours avant l'opération, cinq jours après l'opération (critère d'évaluation principal) et trois mois après l'opération. Des analyses de régression bivariée et multivariée ont été réalisées afin d'identifier les facteurs associés à une DCPO.

Résultats D'un point de vue statistique, les taux de DCPO n'étaient pas différents entre les groupes à cinq jours postopératoires (propofol, 16/72 patients; sévoflurane, 24/ 72 patients; RR, 0,67; IC $95 \%, 0,39$ à 1,15; $P=0,14)$ ou à trois mois postopératoires (propofol, $9 / 60$ patients; sévoflurane, 12/58 patients; RR, 0,73, IC $95 \%, 0,33$ à 1,59; $P=0,42)$. Seuls trois patients par groupe ont manifesté une désaturation peropératoire en oxygène cérébral. L'analyse de régression multivariée a révélé qu'un âge avancé était un prédicteur indépendant de DCPO.

Conclusion Aucune différence significative d'un point de vue statistique n'a été observée en ce qui a trait à l'incidence de DCPO entre les groupes anesthésiés au sévoflurane ou au propofol. La dysfonction cognitive postopératoire était relativement fréquente après une VUP dans les deux groupes. (Numéro d'enregistrement: UMIN 000002826).

Postoperative cognitive dysfunction (POCD) is common following non-cardiac surgery. In a prospective multicentre trial of 1,218 patients undergoing non-cardiac surgery, POCD was present in $26 \%$ of patients one week postoperatively and in $10 \%$ of patients three months postoperatively. ${ }^{1}$ Postoperative cognitive dysfunction is associated with increased mortality ${ }^{2}$ and may lead to a loss of employment and to dependence on social transfer payments, ${ }^{3}$ therefore, it is crucial to identify factors that increase the risk of POCD in order to establish appropriate preventative measures.

Older age appears to be the strongest predictor amongst the number of potential risk factors identified for POCD. ${ }^{4}$ In addition to demographic, clinical, and intraoperative factors, postoperative and hospital-associated factors (e.g., stress, pain, and inflammation) may contribute to the development of POCD. ${ }^{5}$ Recently, Cai et al. reported that POCD in patients receiving inhalation anesthesia was higher than in patients receiving intravenous anesthesia, particularly in inhalation anesthesia patients harbouring the apolipoprotein E \&4 allele associated with Alzheimer's disease. ${ }^{6}$ Thus, choice of anesthesia may be an additional factor influencing the incidence of POCD. Several additional clinical studies have also compared the incidence of POCD in patients undergoing intravenous vs inhalation anesthesia. ${ }^{7-9}$ Nevertheless, these investigations were limited in size $(n=29-101)$, conducted in heterogeneous patient groups anesthetized with a variety of anesthetic agents, and evaluated using different cognitive batteries. Thus, their results may not be applicable to other surgical populations (e.g., lung surgery patients).

One-lung ventilation (OLV) during lung surgery can impair cerebral oxygen balance, and several studies have found that cerebral desaturation increases the risk of POCD following non-cardiac surgery. ${ }^{10-12}$ A previous study reported that cerebral oxygen saturation was higher in the sevoflurane anesthesia group than in the propofol anesthesia group during lung surgery. ${ }^{13}$ Alternatively, a recent review found little evidence for differences in outcomes between inhalation and intravenous anesthesia during lung surgery. ${ }^{14}$ The purpose of this study was to compare the effect of sevoflurane $v s$ propofol anesthesia on postoperative neurocognitive test scores and cerebral oxygenation parameters in a population of patients undergoing lung surgery. The primary outcome was defined as the incidence of POCD on postoperative day five.

\section{Methods}

This prospective randomized patient-blinded single-centre study was approved (February 1, 2007) by the Ethics Committee of Nara Medical University Hospital (Kashihara, Japan; study number 06-043). Written informed consent was obtained from all participants. Study enrolment began after institutional review board approval but before UMIN registration because registration of clinical trials was not required in Japan until 2009, two years after the study was initiated. The government recommended registration of all clinical trials beginning in 2009.

Patients were eligible for inclusion in the study if they were scheduled for elective lung surgery and were 20-85 yr of age. Additional inclusion criteria were American Society of Anesthesiologists physical status I-III, fluency in 
Japanese, ability to read, and absence of serious hearing or visual impairments that would preclude neuropsychological testing. The following exclusion criteria were applied: interstitial lung disease or lung fibrosis, pregnancy or possibility of pregnancy, a history of neurological or mental illness, baseline Mini Mental State Examination (MMSE) score below 24, renal insufficiency (serum creatinine in excess of $1.5 \mathrm{mg} \cdot \mathrm{dL}^{-1}$ ), active liver disease (aspartate aminotransferase in excess of 40 $\mathrm{U} \cdot \mathrm{dL}^{-1}$ ), or documented coagulopathy (prothrombin time $>15 \mathrm{sec}$, activated partial thromboplastin time $>32 \mathrm{sec}$, or platelet count $<150,000 \cdot \mu \mathrm{L}^{-1}$ ).

The patients were randomly allocated to receive either total intravenous anesthesia with propofol or inhalation anesthesia with sevoflurane. A computer-generated blocked randomization list was used to separate patients into two equal treatment groups. Allocation concealment was assured by the use of numbered sealed opaque envelopes. The enrolment of participants and random allocation were conducted independently by different staff members, and the envelopes were drawn by anesthesiologists not involved in this study. The attending surgical anesthesiologists were not blinded to the anesthetic agents. All anesthetic procedures, except cannulation of the jugular vein, were performed by anesthesiologists not involved in this study, and the anesthetic dose was also chosen independently by anesthesiologists not involved in this study. All the operations were performed by the same surgical team.

Cognitive function was assessed two days preoperatively, five days postoperatively, and three months postoperatively using a battery of seven neuropsychological tests: the MMSE, Trail Making Test (Parts A and B), Digit Span (forward and backward), and Grooved Pegboard Test (dominant and non-dominant hands). ${ }^{15,16}$ As described previously, POCD was defined as a decline of $>20 \%$ from preoperative test scores in at least two of seven tests. ${ }^{17}$ Cognitive function was assessed by the same anesthesiologist blinded to group allocation and not involved in intraoperative management. All cognitive tests were performed in the same hospital room.

Before induction of anesthesia, a thoracic epidural catheter was inserted between thoracic 5-6 or 7-8 intervertebral spaces. Beginning before general anesthesia, regional cerebral oxygen saturation was continuously monitored on the left and right sides of the patient's forehead using an INVOS $^{\mathrm{TM}} 5100$ oximeter (Somanetics, Troy, MI, USA). Electrocardiogram, oxygen saturation, invasive arterial pressure, end-tidal carbon dioxide, bladder temperature, and bispectral index (BIS) (Aspect, BIS Monitor A-2000; Nihon Kohden, Tokyo, Japan) were continuously monitored during anesthesia. In the propofol group, anesthesia was initiated with a targetcontrolled infusion (target concentration, 3-4 $\mu \mathrm{g} \cdot \mathrm{mL}^{-1}$ ) of propofol and bolus administration of fentanyl (2.0-2.5 $\left.\mu \mathrm{g} \cdot \mathrm{kg}^{-1}\right)$. In the sevoflurane group, anesthesia was initiated with bolus administration of propofol $\left(1-2 \mathrm{mg} \cdot \mathrm{kg}^{-1}\right)$ and fentanyl (2.0-2.5 $\left.\mu \mathrm{g} \cdot \mathrm{kg}^{-1}\right)$. Rocuronium (0.6-0.9 $\left.\mathrm{mg} \cdot \mathrm{kg}^{-1}\right)$ was administered in both groups to facilitate tracheal intubation.

A left-sided double-lumen endotracheal tube (Portex ${ }^{\circledR}$ Blue Line ${ }^{\circledR}$ Endobronchial Tube; Smith Medical Japan, Tokyo, Japan) was inserted, and the correct position confirmed using a flexible bronchoscope. Anesthesia was maintained with propofol or sevoflurane according to treatment group, plus fentanyl and epidural anesthesia in both groups. The target blood concentration of propofol and expired concentration of sevoflurane were adjusted to keep the BIS score within 40-60. The fraction of inspired oxygen was maintained at 1.0 following anesthesia induction, and the lung protective strategy of low tidal volume and positive end-expiratory pressure (PEEP) was applied during OLV in accordance with the recommended ventilation strategy for lung surgery. ${ }^{18}$ Pressure-controlled ventilation with 4-5 $\mathrm{cm} \mathrm{H}_{2} \mathrm{O}$ PEEP was used for both twolung ventilation and OLV. For two-lung ventilation, the tidal volume was set at $8-10 \mathrm{~mL} \cdot \mathrm{kg}^{-1}$ (predicted body weight), and the respiratory rate was adjusted to maintain arterial partial pressure of carbon dioxide $\left(\mathrm{PaCO}_{2}\right)$ at approximately $40 \mathrm{mmHg}$. For OLV, the tidal volume was set at $5-6 \mathrm{~mL} \cdot \mathrm{kg}^{-1}$ (predicted body weight), and the respiratory rate was set at $10-20$ breaths $\cdot \mathrm{min}^{-1}$. During OLV, peak inspiratory pressure was maintained $<35 \mathrm{~cm}$ $\mathrm{H}_{2} \mathrm{O}$.

To obtain jugular venous blood samples, a 19G long elastic catheter (EVTL-MR 19G $150 \mathrm{~mm}$ RB; Hakko, Tokyo, Japan) was inserted into the right jugular bulb after induction of anesthesia. The position was verified by radiography in the operating room. Arterial and jugular bulb venous blood samples were obtained before OLV, 15 and $60 \mathrm{~min}$ after onset of OLV, and $15 \mathrm{~min}$ after termination of OLV. The following parameters were measured using an ABL 725 blood gas analyzer (Radiometer, Copenhagen, Denmark): arterial oxygen saturation, jugular bulb venous oxygen saturation $\left(\mathrm{SjO}_{2}\right)$, arterial partial pressure of oxygen $\left(\mathrm{PaO}_{2}\right)$, and $\mathrm{PaCO}_{2}$. Cerebral desaturation was defined as 1) an absolute $\mathrm{SjO}_{2}<$ $50 \%$ at any point before OLV, $15 \mathrm{~min}$ after the onset of OLV, 60 min after the onset of OLV, or 15 min after the termination of OLV; or 2) an absolute regional cerebral oxygen saturation $\left(\mathrm{rSO}_{2}\right)<50 \%$ for at least $15 \mathrm{sec}$ at any one of the same measurement time points; or 3) a reduction in $\mathrm{rSO}_{2}$ to $<80 \%$ of baseline for at least $15 \mathrm{sec}$ at any one of the same measurement time points. ${ }^{10,19-21}$ 
Fig. 1 CONSORT flow diagram showing the progress of patients throughout the trial

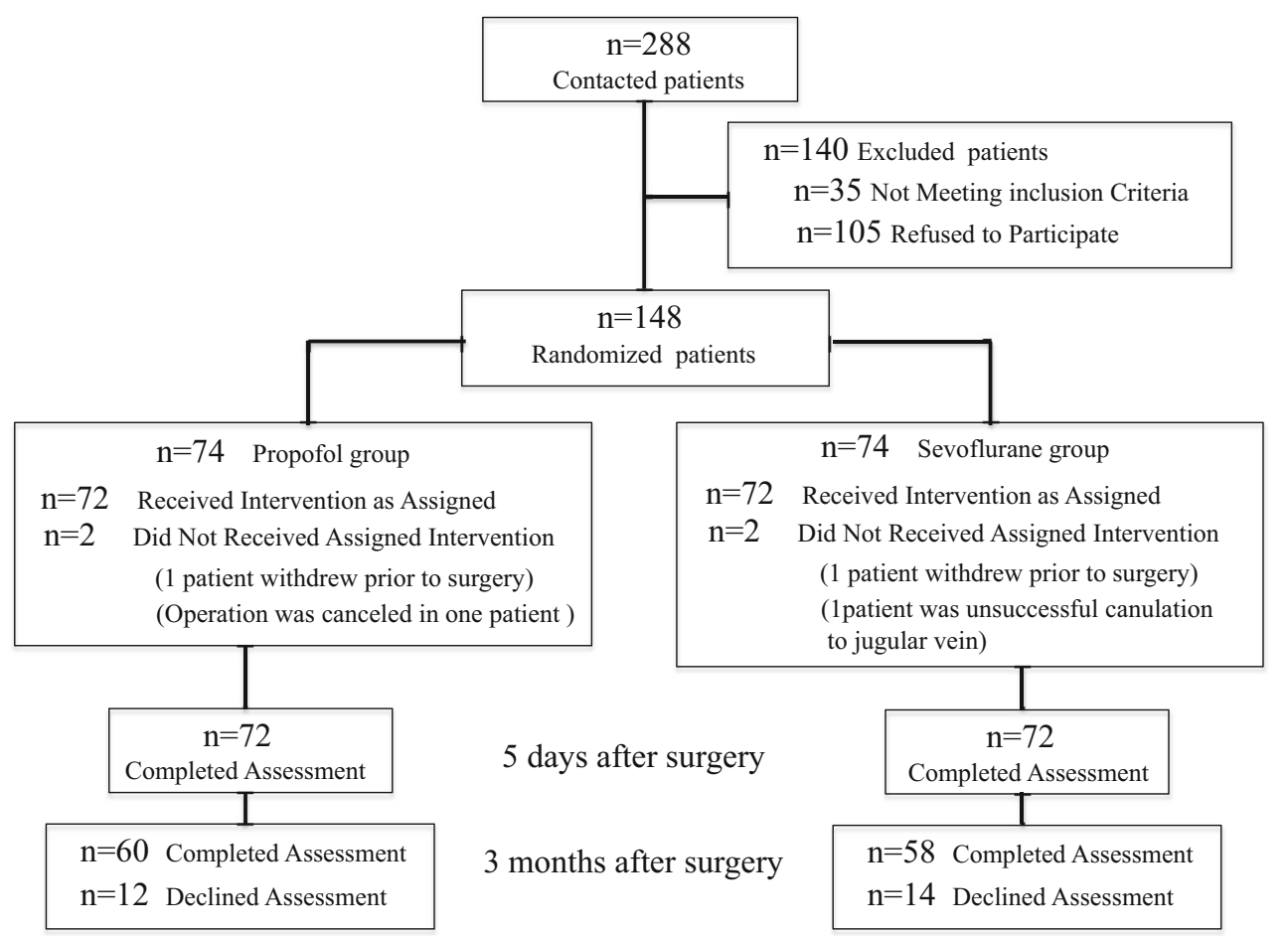

Statistical analysis

The sample size was determined based on our preliminary study evaluating POCD five days after surgery and on a previous study. ${ }^{9}$ We assumed that the incidence of POCD could be as high as $30 \%$ in the sevoflurane group and $10 \%$ in the propofol group. ${ }^{22}$ Considering a $20 \%$ difference in incidence to be clinically significant, 144 patients would be needed to achieve a statistical power of 0.8 with a type I error of 0.05 . To compensate for withdrawals, 148 patients were enrolled in the study.

Bivariable and multivariable regression analyses were used to identify the predictors of POCD. Following identification of candidate factors by univariate regression, all variables with $P<0.2$ were included in the multivariable regression analysis along with age and sex (which were included in the univariate analysis, although not significant).

The statistical software program SPSS $®$ version 11 was used for power analysis (SPSS Inc., Chicago, IL, USA); StatFlex version 6 software was used for univariate and multivariable analyses (Artech Inc., Osaka, Japan), and Prism 6 software was used for other analyses (GraphPad Software, Inc., La Jolla, CA, USA). Dichotomous variables were compared by Chi square or Fisher's exact tests, and depending on the data distribution, results are presented as mean (standard deviation [SD]) or median [interquartile range]. Values of $\mathrm{rSO}_{2}$ and $\mathrm{SjO}_{2}$ were compared between groups by two-way analysis of variance and a post hoc test. All reported $P$ values are two sided.

\section{Results}

From March 2007 to January 2013, we contacted all 288 patients scheduled for elective lung surgery at Nara Medical University, Nara, Japan. One hundred five patients refused to participate, and 35 patients either did not meet all the inclusion criteria or met at least one of the exclusion criteria. Consequently, 148 patients were enrolled in this study and randomized equally to the sevoflurane or propofol group. Two patients who were initially accepted in the sevoflurane group were excluded from the analysis. One withdrew prior to surgery, and the other was excluded because of unsuccessful jugular vein cannulation during surgery. Two patients in the propofol group were also excluded. One withdrew prior to surgery, and surgery for the other patient was cancelled. Data for 144 patients were included in the final analysis (Fig. 1).

Patient demographics, baseline clinical characteristics, and surgical data are shown in Table 1. The durations of anesthesia and surgery were longer in the propofol group than in the sevoflurane group, and the median dose of intraoperative fentanyl was higher in the propofol group than in the sevoflurane group. Hemodynamic variables, BIS values, bladder temperature, and arterial blood gas parameters are presented in Table A (available as Electronic Supplementary Material). Postoperative tests for the five-day time point were conducted on postoperative day 5 (range, 5-5), while the tests for the three-month time point were conducted on day 88 (range, 
Table 1 Baseline characteristics and intra-operative data of the study patients

\begin{tabular}{lll}
\hline & Propofol $(n=72)$ & Sevoflurane $(n=72)$ \\
\hline Age $(\mathrm{yr})$ & $69[63-73]$ & $72[63-72]$ \\
Sex $(\mathrm{F} / \mathrm{M})$ & $23 / 48$ & $33 / 39$ \\
BMI $\left(\mathrm{kg} \cdot \mathrm{m}^{-2}\right)$ & $23.3(3.1)$ & $22.7(3.5)$ \\
ASA grade (I/II/III) & $25 / 42 / 5$ & $29 / 40 / 3$ \\
Duration of anesthesia & $284(82)$ & $254(62)$ \\
$\quad(\mathrm{min})$ & & \\
Duration of surgery (min) & $195(77)$ & $167(70)$ \\
Intraoperative fentanyl $(\mu \mathrm{g})$ & $300[250-400]$ & $300[200-300]$ \\
Years of education $(\mathrm{yr})$ & $12[12-13]$ & $12[9-12]$ \\
Baseline MMSE score & $30[29-30]$ & $30[28-30]$ \\
\hline
\end{tabular}

Data are presented as median [interquartile range] or mean (standard deviation) as indicated

ASA = American Society of Anesthesiologists; BMI = body mass index; MMSE $=$ Mini Mental State Examination

78-94). All patients had uneventful intraoperative and postoperative courses.

There was no significant difference in the incidence of POCD between the propofol and sevoflurane groups five days postoperatively [16 of 72 (22\%) patients $v s 24$ of 72 (33\%) patients, respectively; risk ratio (RR), 0.67; 95\% confidence interval (CI), 0.39 to $1.15 ; P=0.14]$. Twentysix of 144 patients were lost to follow-up three months postoperatively (12 patients in the propofol group and 14 patients in the sevoflurane group); consequently, 118 patients were analyzed three months postoperatively (Fig. 1). Again, there was no significant difference in the incidence of POCD between the propofol and sevoflurane groups [ 9 of $60(15 \%)$ patients vs 12 of $58(21 \%)$ patients, respectively; RR, $0.73 ; 95 \% \mathrm{CI}, 0.33$ to $1.6 ; P=0.42$ ). In the propofol group, three of 16 patients who showed POCD five days postoperatively were lost to follow-up three months postoperatively. Of the 13 remaining patients, four still showed POCD at three months postoperatively. In the sevoflurane group, four of 24 patients who showed POCD five days postoperatively were lost to follow-up three months postoperatively. Of the 20 remaining patients, eight still showed POCD at three months postoperatively. The median scores on all seven cognitive tests are shown in Table 2 for both anesthesia groups.

There were no significant differences in $\mathrm{PaO}_{2}$ and $\mathrm{PaCO}_{2}$ values between the two groups at any measurement

Table 2 Cognitive test results of propofol and sevoflurane groups at baseline, five days postoperatively, and three months postoperatively

\begin{tabular}{|c|c|c|c|}
\hline & Baseline & 5 days after surgery & 3 months after surgery \\
\hline \multicolumn{4}{|l|}{ MMSE } \\
\hline propofol & $30[29-30]$ & $30[27-30]$ & $30[29-30]$ \\
\hline sevoflurane & $30[28-30]$ & $30[28-30]$ & $30[29-30]$ \\
\hline \multicolumn{4}{|l|}{ TMT A (sec) } \\
\hline propofol & $42[32-64]$ & $44[32-57]$ & $43[33-56]$ \\
\hline sevoflurane & $43[30-59]$ & $41[29-57]$ & $45[32-57]$ \\
\hline \multicolumn{4}{|l|}{ TMT B (sec) } \\
\hline propofol & $100[78-140]$ & 91 [72-117] & $93[71-121]$ \\
\hline sevoflurane & $107[76-140]$ & $104[81-155]$ & 108 [73-133] \\
\hline \multicolumn{4}{|c|}{ Digit Span Forward } \\
\hline propofol & $7[6-8]$ & $7[6-8]$ & $7[5-8]$ \\
\hline sevoflurane & $6[5-7]$ & $6[5-7]$ & $6[5-8]$ \\
\hline \multicolumn{4}{|c|}{ Digit Span Backward } \\
\hline propofol & $4[3-5]$ & $4[3-6]$ & $4[3.5-5.5]$ \\
\hline sevoflurane & $4[3-5]$ & $4[3-5]$ & $4[4-5.5]$ \\
\hline \multicolumn{4}{|c|}{ Pegboard (dominant hand) (sec) } \\
\hline propofol & $78[65-94]$ & $81[71-98]$ & $75[64-91]$ \\
\hline sevoflurane & 82 [70-102] & 82 [71-103] & $73[65-92]$ \\
\hline \multicolumn{4}{|c|}{ Pegboard (non-dominant hand) (sec) } \\
\hline propofol & 85 [73-106] & 91 [77-112] & 80 [70-105] \\
\hline sevoflurane & 94 [76-123] & $95[80-121]$ & $91[76-105]$ \\
\hline
\end{tabular}

POCD incidence: 16 of 72 in the propofol group, 24 of 72 in the sevoflurane group (RR, $0.67 ; 95 \% \mathrm{CI}, 0.39$ to $1.15 ; P=0.14$ ) at 5 days after surgery; 9 of 60 in the propofol group, 12 of 58 in the sevoflurane group (RR, $0.73 ; 95 \%$ CI, 0.33 to $1.59 ; P=0.42$ ) at three months after surgery. Data are presented as median [interquartile range]

MMSE = Mini Mental State Examination; TMT A = Trail Making Test Part A; TMT B = Trail Making Test Part B 
Fig. 2 Changes in $\mathrm{rSO}_{2}$ and $\mathrm{SjO}_{2}$ before, during, and following one-lung ventilation $(\mathrm{OLV})$. Time $0=$ awake; Time 1 = before OLV; Time $2=15 \mathrm{~min}$ after OLV onset; Time $3=60$ min after OLV onset; and Time $4=15$ min after OLV termination. $\mathrm{POCD}=$ postoperative cognitive dysfunction; $\mathrm{rSO}_{2}=$ regional cerebral oxygen saturation; $\mathrm{SjO}_{2}=$ jugular bulb venous oxygen saturation
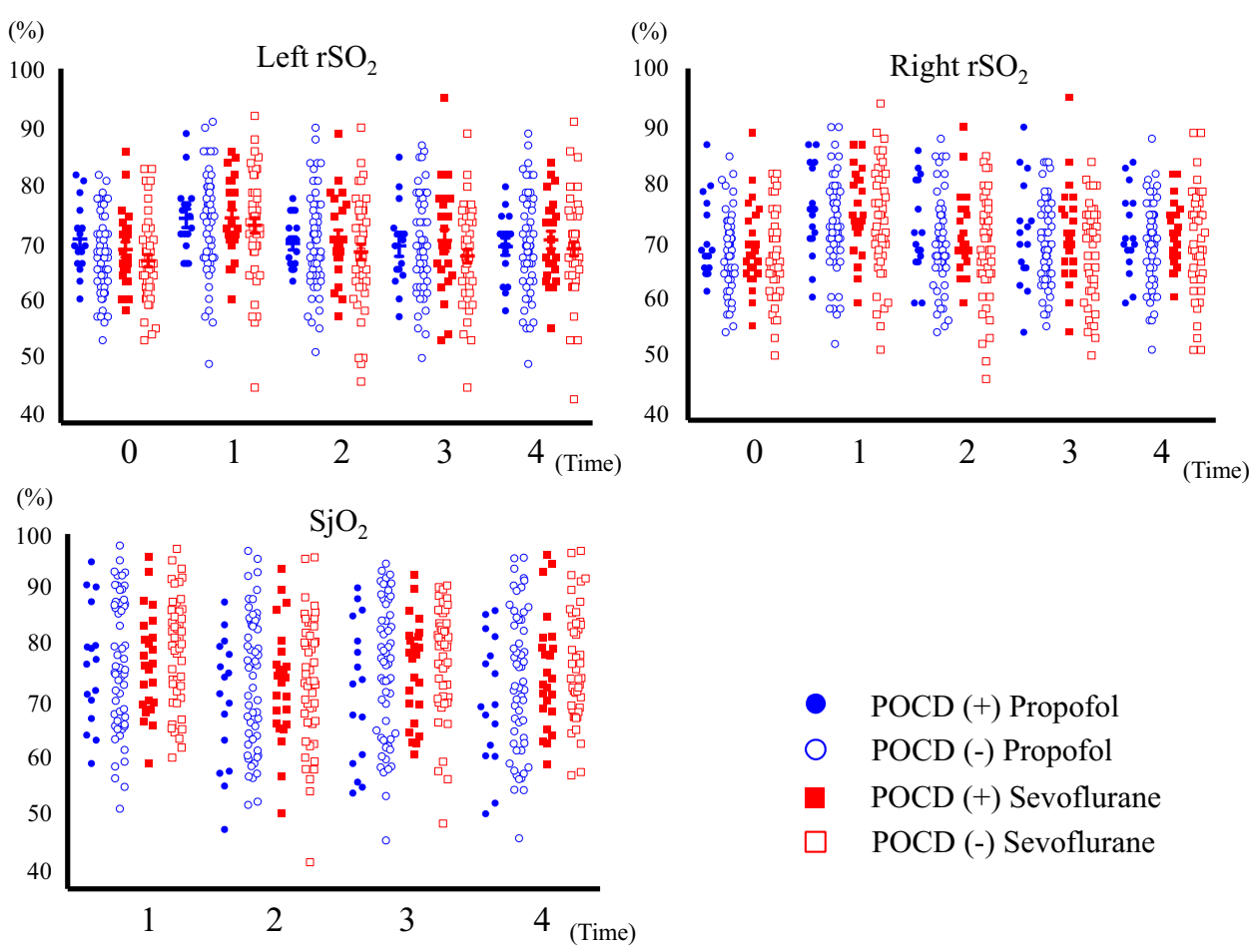

- POCD (+) Propofol

POCD (-) Propofol

- POCD $(+)$ Sevoflurane

POCD (-) Sevoflurane time point (Table A, available as Electronic Supplementary Material). Similarly, there were no significant differences in $\mathrm{rSO}_{2}$ and $\mathrm{SjO}_{2}$ values at the defined measurement time points among the POCD-positive propofol, POCD-negative propofol, POCD-positive sevoflurane, and POCD-negative sevoflurane subgroups -as defined five days postoperatively (Fig. 2). The incidence of cerebral desaturation $\left(\mathrm{rSO}_{2}\right.$ or $\mathrm{SjO}_{2}<50 \%$ or a reduction in $\mathrm{rSO}_{2}$ to $<80 \%$ of baseline) did not differ between the propofol and sevoflurane groups (three patients or $4 \% v s$ three patients or $4 \%$, respectively; RR, $1.0 ; 95 \%$ CI, 0.21 to $4.8 ; P=1.0$ ).

On univariate analysis, age was a significant factor associated with POCD after lung surgery. Multivariable regression analysis revealed age as the only major independent predictor of POCD (Table 3).

\section{Discussion}

There is still considerable debate over whether specific anesthetics increase the risk of POCD in non-cardiac surgery patients. Moreover, postoperative cognitive function of lung surgery patients may be particularly sensitive to certain anesthetics if the substances influence intraoperative cerebral oxygen balance. A reduction in POCD was identified five days following surgery was noted among patients receiving propofol (RR, 0.67; 95\% CI, 0.39 to 1.15). A difference of this size might be of clinical significance but did not reach statistical significance. The incidence of cerebral oxygen desaturation (RR, $1.00 ; 95 \% \mathrm{CI}, 0.21$ to $4.79 ; P=1.00$ ) was similar in propofol- or sevoflurane-based anesthesia groups. At three months, POCD was relatively frequent in both groups [21 of $118(18 \%)]$, underscoring the potential for long-lasting POCD in non-cardiac surgery patients. Nevertheless, we found no corresponding difference in POCD between anesthetic treatment groups (RR, 0.73; 95\% CI, 0.33 to $1.59 ; \quad P=0.42$ ) three months postoperatively. Age was the predominant influence on the risk of POCD, which is consistent with several previous studies.

Postoperative cognitive dysfunction is associated with greater mortality after non-cardiac surgery. ${ }^{2}$ Although several mechanisms have been proposed, ${ }^{5}$ POCD etiology is still unclear. According to the report of the Second International Perioperative Neurotoxicity Workshop, there is still considerable controversy over whether specific anesthetic agents increase the risk of POCD. ${ }^{23,24}$ Nonetheless, POCD is relatively common after general anesthesia. $^{25}$ Several prospective ${ }^{7-9}$ and retrospective ${ }^{6}$ studies have compared the incidence of POCD after intravenous $v s$ inhalation anesthesia. Enlund et al. compared the incidence of POCD in 29 orthognathic surgery patients anesthetized with propofol $v s$ isoflurane, ${ }^{7}$ whereas Rohan et al. compared the incidence of POCD in 30 patients undergoing cystoscopic or hysteroscopic surgery and anesthetized with propofol vs sevoflurane. ${ }^{8}$ Both studies reported no significant differences in the 
Table 3 Bivariable and multivariable regression analyses

\begin{tabular}{|c|c|c|c|c|}
\hline \multirow[b]{2}{*}{ Variable } & \multicolumn{2}{|l|}{5 days after surgery } & \multicolumn{2}{|l|}{3 months after surgery } \\
\hline & Odds Ratio $(95 \% \mathrm{CI})$ & $P$ value & Odds Ratio $(95 \% \mathrm{CI})$ & $P$ value \\
\hline \multicolumn{5}{|l|}{ Bivariable predictors } \\
\hline Age & $1.08(1.03$ to 1.13$)$ & 0.003 & $1.07(1.02$ to 1.13$)$ & 0.01 \\
\hline Sex (male vs female) & $1.23(0.59$ to 2.6$)$ & 0.58 & $1.64(0.74$ to 3.6$)$ & 0.23 \\
\hline Anesthetic agent (propofol vs sevoflurane) & $1.75(0.83$ to 3.7$)$ & 0.14 & $1.97(0.88$ to 4.4$)$ & 0.10 \\
\hline Duration of anesthesia (hr) & $1.1(0.82$ to 1.48$)$ & 0.53 & 1.03 (0.75 to 1.42$)$ & 0.86 \\
\hline Intraoperative fentanyl dose $\left(\mu \mathrm{g} \cdot \mathrm{kg}^{-1}\right)$ & $0.97(0.67$ to 1.41$)$ & 0.88 & 0.98 (0.66 to 1.46$)$ & 0.92 \\
\hline Years of education ( $>9$ years $v s<10$ years) & $0.68(0.33$ to 1.41$)$ & 0.30 & $1.46(0.58$ to 3.7$)$ & 0.42 \\
\hline Cerebral desaturation (No vs Yes) & $0.51(0.06$ to 4.5$)$ & 0.54 & $0.66(0.07$ to 6.1$)$ & 0.71 \\
\hline \multicolumn{5}{|l|}{ Multivariable predictors } \\
\hline Age & $1.08(1.03$ to 1.13$)$ & 0.003 & 1.08 (1.03 to 1.14$)$ & 0.004 \\
\hline Sex (male vs female) & $1.6(0.71$ to 3.6$)$ & 0.26 & $2.26(0.93$ to 5.5$)$ & 0.07 \\
\hline Anesthetic agent (propofol vs sevoflurane) & $1.44(0.66$ to 3.14$)$ & 0.36 & $1.74(0.75$ to 4.0$)$ & 0.20 \\
\hline
\end{tabular}

Following identification in bivariable regression analysis, variables with values of $P<0.2$ were included in multivariable regression. CI $=$ confidence interval

incidence of POCD between the intravenous and inhalation anesthetic groups. Nevertheless, both patient groups were likely too small to detect a modest but clinically relevant difference. In contrast, the current study of 144 patients was designed to detect a difference in this range, even in cases of subtle deficits. To accomplish this objective, we used multiple cognitive tests probing different domains, including MMSE (screening), Trail Making Test Part A (attention), Trail Making Test Part B (executive function), Digit Span Test (verbal working memory), and Grooved Pegboard Test (motor function). According to a consensus statement, the Rey Auditory Verbal Learning Test, Trail Making Test A/B, and Grooved Pegboard Test are recommended as core components of a comprehensive neuropsychological battery. ${ }^{15}$ In our preliminary studies, we included the Rey Auditory Verbal Learning Test; however, it was excluded from the main trial as it took a long time to complete and was stressful for some postoperative patients.

We focused on lung surgery because, according to the latest report of the International Agency for Research on Cancer, lung cancer is currently the most commonly diagnosed cancer worldwide, and lung cancer surgeries are increasing, even among the elderly. Hemmerling et al. reported that lung surgery with OLV is associated with a substantial decrease in cerebral oxygen saturation in the majority of patients. ${ }^{26}$ Likewise, Kazan et al. reported that $82 \%$ of 50 patients undergoing thoracic surgery exhibited a reduction in cerebral oxygen saturation of $>15 \%$ from their preoperative baseline. ${ }^{27}$ Iwata et al. reported that the incidence of cerebral desaturation during OLV for lung surgery was higher when using propofol-based anesthesia vs sevoflurane-based anesthesia. ${ }^{13}$ In addition, previous studies have shown that intraoperative regional cerebral desaturation is associated with lower postoperative MMSE scores in lung surgery patients. ${ }^{12,28}$ Therefore, cerebral desaturation is considered a likely causative factor for POCD. A previous report ${ }^{13}$ showed that cerebral desaturation was related to choice of anesthetic in OLV. Contrary to the results of Iwata et al., we found no significant difference in the incidence of cerebral desaturation between the propofol and sevoflurane anesthesia groups. Nevertheless, desaturation was rare in both groups, possibly because we applied the lung protective strategy with $100 \%$ oxygen during OLV. In fact, in the aforementioned study, $\mathrm{SpO}_{2}$ values were maintained at around 95\%, which is relatively low compared with our results. The $\mathrm{PaCO}_{2}$ values were also slightly lower compared with our patients. As a result, absolute $\mathrm{SjO}_{2}$ values in our propofol group were relatively higher than in their study; consequently, maintaining high cerebral oxygen saturation may offset any differences due to the choice of anesthetic. As there was a low incidence of desaturation in both groups, we could not clarify the correlation between cognitive dysfunction and cerebral desaturation.

There are several limitations to this study. First, it was conducted at a single university hospital, thereby introducing the possibility of bias in patient selection and possibly limiting the general applicability of our results. ${ }^{29}$ Second, we did not use a double dummy blinding method in the selection of anesthetic agents because it was not feasible to reliably blind anesthesiologists in a clinical setting. Nevertheless, all the anesthetic procedures, except 
for cannulation of the jugular vein, were performed by anesthesiologists who were not involved in this study. In addition, the dose of drug within the protocol was also decided independently by non-participant anesthesiologists. Third, the mean duration of anesthesia and surgery was significantly longer in the propofol group than in the sevoflurane group. Nevertheless, because the mean difference in duration was only $30 \mathrm{~min}$, we suggest that there was minimal influence on the neurocognitive test scores. Finally, patients with severe lung disease, such as interstitial lung disease or lung fibrosis, were excluded from the study. The incidence of desaturation may be higher in such patients, which could alter the incidence of POCD and possibly unmask a differential influence of anesthetics on cognitive function.

In conclusion, there was no statistically significant difference in the incidence of POCD between propofoland sevoflurane-based anesthesia groups following lung surgery. The incidence of cerebral desaturation during surgery did not differ between the anesthesia groups under the lung protective respiratory strategy. Larger randomized-controlled trials are required to determine if choice of anesthesia influences cognitive test results in the early postoperative period.

Funding This work was supported by the Department of Anesthesiology, Nara Medical University, Kashihara, Nara, Japan.

Conflicts of interest None declared.

Author contributions Junji Egawa, Masahiko Kawaguchi, Hitoshi Furuya, Satoki Inoue, and Tadashi Nishiwada contributed to the study design. Junji Egawa contributed to patient recruitment, study conduct, and manuscript preparation. Junji Egawa, Satoki Inoue, and Tadashi Nishiwada contributed to data analysis. Masahiko Kawaguchi and Hitoshi Furuya contributed to data interpretation. Masahiko Kawaguchi, Hitoshi Furuya, Takashi Tojo, Michitaka Kimura, Takeshi Kawaguchi, and Shigeki Taniguchi contributed to manuscript review. Satoki Inoue and Tadashi Nishiwada contributed to blood sample and hemodynamic data collection. Takashi Tojo, Michitaka Kimura, Takeshi Kawaguchi, and Shigeki Taniguchi obtained informed consent from patients and contributed to postoperative care. Masahiko Kawaguchi was the principal investigator.

Editorial responsibility This submission was handled by Dr. Gregory L. Bryson, Deputy Editor-in-Chief, Canadian Journal of Anesthesia.

\section{References}

1. Moller JT, Cluitmans P, Rasmussen LS, et al. Long-term postoperative cognitive dysfunction in the elderly ISPOCD1 study. ISPOCD investigators. International Study of PostOperative Cognitive Dysfunction. Lancet 1998; 351: 857-61.
2. Monk TG, Weldon BC, Garvan CW, et al. Predictors of cognitive dysfunction after major noncardiac surgery. Anesthesiology 2008; 108: 18-30.

3. Steinmetz J, Christensen KB, Lund T, Lohse N, Rasmussen LS, ISPOCD Group. Long-term consequences of postoperative cognitive dysfunction. Anesthesiology 2009; 110: 548-55.

4. Burkhart CS, Steiner LA. Can postoperative cognitive dysfunction be avoided? Hosp Pract 1995; 2012(40): 214-23.

5. Krenk L, Rasmussen $L S$, Kehlet $H$. New insights into the pathophysiology of postoperative cognitive dysfunction. Acta Anaesthesiol Scand 2010; 54: 951-6.

6. Cai $Y, H u H$, Liu P, et al. Association between the apolipoprotein E4 and postoperative cognitive dysfunction in elderly patients undergoing intravenous anesthesia and inhalation anesthesia. Anesthesiology 2012; 116: 84-93.

7. Enlund $M$, Mentell $O$, Flenninger A, Horneman $G$, Ronquist $G$. Evidence of cerebral dysfunction associated with isoflurane- or propofol based anaesthesia for orthognathic surgery, as assessed by biochemical and neuropsychological methods. Ups J Med Sci 1998; 103: 43-59.

8. Rohan D, Buggy DJ, Crowley S, et al. Increased incidence of postoperative cognitive dysfunction $24 \mathrm{hr}$ after minor surgery in the elderly. Can J Anesth 2005; 52: 137-42.

9. Hocker J, Stapelfeldt C, Leiendecker J, et al. Postoperative neurocognitive dysfunction in elderly patients after xenon versus propofol anesthesia for major noncardiac surgery: a doubleblinded randomized controlled pilot study. Anesthesiology 2009; 110: 1068-76.

10. Casati A, Fanelli G, Pietropaoli P, et al. Continuous monitoring of cerebral oxygen saturation in elderly patients undergoing major abdominal surgery minimizes brain exposure to potential hypoxia. Anesth Analg 2005; 101: 740-7.

11. Ballard C, Jones E, Gauge N, et al. Optimised anaesthesia to reduce post operative cognitive decline (POCD) in older patients undergoing elective surgery, a randomised controlled trial. PLoS One 2012; 7: e37410.

12. Tang L, Kazan R, Taddei R, Zaouter C, Cyr S, Hemmerling TM. Reduced cerebral oxygen saturation during thoracic surgery predicts early postoperative cognitive dysfunction. Br J Anaesth 2012; 108: 623-9.

13. Iwata $M$, Inoue $S$, Kawaguchi $M$, et al. Jugular bulb venous oxygen saturation during one-lung ventilation under sevofluraneor propofol-based anesthesia for lung surgery. J Cardiothorac Vasc Anesth 2008; 22: 71-6.

14. Modolo NS, Modolo MP, Marton MA, et al. Intravenous versus inhalation anaesthesia for one-lung ventilation. Cochrane Database Syst Rev 2013; 7: CD006313.

15. Murkin JM, Newman SP, Stump DA, Blumenthal JA. Statement of consensus on assessment of neurobehavioral outcomes after cardiac surgery. Ann Thorac Surg 1995; 59: 1289-95.

16. Rundshagen I. Postoperative cognitive dysfunction. Dtsch Arztebl Int 2014; 111: 119-25.

17. Grichnik KP, Ijsselmuiden AJ, D'Amico TA, et al. Cognitive decline after major noncardiac operations: a preliminary prospective study. Ann Thorac Surg 1999; 68: 1786-91.

18. Slinger $P$. Pro: low tidal volume is indicated during one-lung ventilation. Anesth Analg 2006; 103: 268-70.

19. Robertson CS, Gopinath SP, Goodman JC, Contant CF, Valadka $A B$, Narayan RK. SjvO2 monitoring in head-injured patients. J Neurotrauma 1995; 12: 891-6.

20. Baris RR, Israel AL, Amory DW, Benni P. Regional cerebral oxygenation during cardiopulmonary bypass. Perfusion 1995; 10 : 245-8.

21. Samra SK, Dy EA, Welch K, Dorje P, Zelenock GB, Stanley JC. Evaluation of a cerebral oximeter as a monitor of cerebral 
ischemia during carotid endarterectomy. Anesthesiology 2000; 93: 964-70.

22. Royse CF, Andrews DT, Newman SN, et al. The influence of propofol or desflurane on postoperative cognitive dysfunction in patients undergoing coronary artery bypass surgery. Anaesthesia 2011; 66: 455-64.

23. Crosby G, Culley DJ. Surgery and anesthesia: healing the body but harming the brain? Anesth Analg 2011; 112: 999-1001.

24. Eckenhoff $R G$. Second International Perioperative Neurotoxicity Workshop Summary. Anesth Analg 2011; 112: 1253-4.

25. Rasmussen LS, Johnson T, Kuipers HM, et al; ISPOCD2 (International Study of Postoperative Cognitive Dysfunction) Investigators. Does anaesthesia cause postoperative cognitive dysfunction? A randomised study of regional versus general anaesthesia in 438 elderly patients. Acta Anaesthesiol Scand 2003; 47: 260-6.
26. Hemmerling TM, Bluteau MC, Kazan R, Bracco D. Significant decrease of cerebral oxygen saturation during single-lung ventilation measured using absolute oximetry. $\mathrm{Br} \mathrm{J}$ Anaesth 2008; 101: 870-5.

27. Kazan R, Bracco D, Hemmerling TM. Reduced cerebral oxygen saturation measured by absolute cerebral oximetry during thoracic surgery correlates with postoperative complications. $\mathrm{Br}$ J Anaesth 2009; 103: 811-6.

28. Suehiro $K$, Okutai $R$. Duration of cerebral desaturation time during single-lung ventilation correlates with mini mental state examination score. J Anesth 2011; 25: 345-9.

29. Bellomo R, Warrillow SJ, Reade MC. Why we should be wary of single-center trials. Crit Care Med 2009; 37: 3114-9. 